\title{
CORONARY ARTERY DISEASE ALTERS VENTRICULAR REPOLARIZATION DYNAMICS IN TYPE 2 DIABETES
}

Bojan Vrtovec, MD, PhD; Matjaz Sinkovec, MD, PhD; Vito Starc, MD, PhD; Branislav Radovancevic, MD; Todd T. Schlegel, MD

From the Division of Cardiology, Ljubljana University Medical Center, Ljubljana, Slovenia (B.V., M. S.); Institute of Physiology, University of Ljubljana, Ljubljana , Slovenia (V.S.); Division of Cardiopulmonary Transplantation, Texas Heart Institute, Houston, TX, USA (B.R.); and NASA Johnson Space Center, Houston, TX, USA (T.T.S.)

Running title: Ventricular repolarization and diabetes

Correspondence to: Bojan Vrtovec, MD, PhD Division of Cardiology, Ljubljana University Medical Center, Ljubljana, Slovenia. MC 1000, Ljubljana. Telephone: (3861)522-2844; FAX; (3861)230-2828; E-mail: bojan.vrtovec@mf.uni-lj.si 


\begin{abstract}
Background. Ventricular repolarization dynamics (VRD) is an important predictor of outcome in diabetes. We examined the potential impact of coronary artery disease (CAD) on VRD in type 2 diabetic patients.

Methods We recorded 5-min high-resolution resting electrocardiograms (ECG) in 38 diabetic patients undergoing elective cotonary angiography, and in 38 age- and gender- matched apparently healthy subjects (Controls). Using leads I and II, time-domain indices of VRD were calculated. Coronary angiography was regarded as positive if a $\geq 50 \%$ stenosis was found.

Results Angiography was positive in 21 diabetic patients (55\%). Patients with CAD had a significantly higher degree of VRD than Controls (SDNN(QT): $15.81 \pm 7.22 \mathrm{~ms}$ vs. $8.94 \pm 6.04$ $\mathrm{ms} ; P<0.001, \mathrm{rMSSD}(\mathrm{QT}): 21.02 \pm 7.07 \mathrm{~ms}$ vs. $11.18 \pm 7.45 \mathrm{~ms} ; P<0.001$ ). VRD in diabetic patients with negatie angiograms did not differ from VRD in Controls (SDNN(QT): $8.94 \pm 6.04 \mathrm{~ms}$ vs. $7.44 \pm 5.72 \mathrm{~ms} ; P=0.67, \mathrm{rMSSD}(\mathrm{QT}): 11.18 \pm 7.45 \mathrm{~ms}$ vs. $10.22 \pm 5.35 \mathrm{~ms}$; $P=0.82$ ).
\end{abstract}

Conclusions CAD increases VRD in patients with type 2 diabetes. Therefore, changes in ventricular repolarization in diabetic patients may be due to silent $\mathrm{CAD}$ rather than to diabetes per se.

Key words: diabetes, coronary artery disease, ventricular repolarization, QT interval 


\section{Background}

Cardiovascular disease represents the leading cause of death in patients with type 2 diabetes ${ }^{1}$. Stroke and cardiac death can be predicted in these patients by various measures of ventricular repolarization ${ }^{2,3}$. Mechanisms which may influence ventricular repolarization in type 2 diabetes include autonomic nervous system abnormalities ${ }^{4}$, impaired insulin sensitivity, alterations of glucose homeostasis ${ }^{5}$, and coronary artery disease (CAD). Although several studies have demonstrated that $\mathrm{CAD}$ alters ventricular repolarization in non-diabetic subjects $^{6,7}$, its impact on ventricular repolarization in diabetic patients remains unclear. This study was performed to better define the pathophysiologic background of repolarization abnormalities and the impact of CAD on ventricular repolarization dynamics (VRD) in patients with type 2 diabetes.

\section{Patient population and methods}

We performed a double-blind, case-control study in 38 diet-treated patients with type 2 diabetes who underwent elective coronary angiography due to symptoms consistent with angina pectoris (Study group). The Control group included 38 age- and gender-matched, subjects without history of diabetes, with normal plasma glucose levels, and apparently healthy. Patients with hepatic or renal dysfunction, history of heart failure, or cerebrovascular disease were not included in the study. Likewise, patients treated with medications which may alter glucose homeostasis or ventricular repolarization, including thiazides, corticosteroids, phenothiazines, estrogens, sympathomimetics, type I and type II antiarrhythmic drugs, were not studied. The study protocol was approved by the National Ethics Committee, and all patients granted their informed consent prior to entering the study.

In all subjects, a 5-min high resolution 12-lead electrocardiogram (ECG) was recorded at the time of enrollment. In case of atrial, ventricular ectopy or inadequate signal 
quality, the procedure was repeated. The ECG recordings were stored in a computer database and analyzed by an independent observer, who was blinded to the presence of CAD and diabetes mellitus.

\section{Cardiac Catheterization Technique}

In all patients from the Study group, cardiac catheterization was performed within $6 \mathrm{~h}$ after ECG recordings. Coronary angiography was performed using standard techniques. Stenotic lesions were graded subjectively by visual consensus of at least 2 experienced observers on an ordinal scale of $0 \%, 25 \%, 50 \%, 75 \%, 95 \%$, or $100 \%$. The catheterization result was regarded as positive if the coronary angiogram showed $\geq 50 \%$ stenosis in $\geq 1$ major coronary artery.

\section{Analysis of Ventricular Repolarization Dynamics}

The analysis of VRD was performed with a computer-assisted beat-to-beat algorithm ${ }^{7}$. First, the template beat was constructed by averaging all beats based on superposition of the ECG signal according to the triggering point on the $\mathrm{R}$ wave. Then, the operator manually defined the analysis window ranging from end of QRS complex to the end of QT interval on the template signal. The $T$-wave of any beat of the 5 -min recording was shifted to achieve the best fit with the template in the analysis window. For each beat, an error function was defined as the sum of the squared differences between the template and the incoming beat. QT interval duration of each beat was calculated with summation of template QT interval duration and error function parameter. Time-domain measures of VRD and heart rate variability were calculated according to the recommendations of the Task Force of the European Society of Cardiology and the North American Society of Pacing and Electrophysiology ${ }^{8}$

\section{Statistical Analysis}

Continuous variables were expressed as means $\pm \mathrm{SD}$. The differences between the groups 
were analyzed with 1-factor analysis of variance. The comparisons of categorical variables were performed with a chi-square test. The parameters approaching statistical significance on univariate analyses were included in a multivariate model for prediction of increased VRD in diabetic subjects. $P$ values $<0.05$ were considered significant.

\section{Results}

\section{Patient Characteristics}

The baseline characteristics of the the Study and Control groups are presented in Table 1. The two groups did not differ with regards to age, gender, laboratory test results, heart rate, and prevalence of hypertension.

\section{Ventricular Repolarization Dynamics and Diabetes}

When compared to Controls, patients from the Study group had significantly higher values of VRD (SDNN(QT): $12.73 \pm 6.69 \mathrm{~ms}$ in the Study group vs. $7.44 \pm 5.72 \mathrm{~ms}$ in Controls, $P=0.01$; $\operatorname{rMSSD}(\mathrm{QT}): 16.62 \pm 7.24 \mathrm{~ms}$ in the Study group vs. $10.22 \pm 5.35 \mathrm{~ms}$ in Controls, $P=0.01$ ). However, the two groups did not differ with respect to heart rate variability parameters (SDNN(RR): $45.7 \pm 6.9 \mathrm{~ms}$ in the Study group vs. $46.0 \pm 5.2 \mathrm{~ms}$ in Controls, $P=0.77$, rMSSD(RR): $41.6 \pm 6.3 \mathrm{~ms}$ in the Study group vs. $40.2 \pm 7.5 \mathrm{~ms}$ in Controls, $P=0.82$ ).

\section{Ventricular Repolarization Dynamics and Coronary Artery Disease}

Coronary angiography was positive in 21 diabetic patients (55\%). Patients with diabetes and CAD displayed a higher degree of VRD than diabetic patients with normal angiograms, and subjects from the Control group (Figure 1). No difference in VRD between diabetic patients with normal angiograms and the subjects from the Control group was found.

Multivariate Analysis for Prediction of Increased Ventricular Repolarization Dynamics in Diabetes 
Increased $\mathrm{VRD}$, defined as either $\mathrm{SNDD}(\mathrm{QT})>12 \mathrm{~ms}$, or $\mathrm{rMSSD}(\mathrm{QT})>16 \mathrm{~ms}$, was present in 27 diabetic patients $(71 \%)$. The presence of CAD was the only independent predictor of increased VRD in our study (Table 2).

\section{Discussion}

The results of this study demonstrate that patients with type 2 diabetes have a higher degree of VRD than healthy subjects. Changes in VRD in diabetics were strongly related to the presence of CAD. We quantified VRD in diabetic patients using beat-to-beat QT interval variability measurements. This relatively new technique is highly reproducible, and is a reliable marker of ventricular electrical abnormalities, perhaps associated with a higher risk of life-threatening arrhythmias ${ }^{9}$. The abnormal VRD present in over $70 \%$ of our diabetic patients is consistent with previous observations, ${ }^{1}$ and suggests a high prevalence of ventricular repolarization abnormalities in type 2 diabetes.

Although diabetics are at increased risk of sudden cardiac death ${ }^{10}$, the mechanisms underlying such death remain poorly defined. Cardiac autonomic neuropathy is an important disorder which can alter ventricular repolarization and lead to increased sudden cardiac death in this patient population ${ }^{11}$. We found no differences in heart rate variability indices between diabetics and Controls, suggesting a relatively mild degree of autonomic nervous system impairment in our diabetic patients. Therefore, although a role played by the autonomic nervous system cannot be excluded, it seems unlikely to be responsible for the differences in VRD observed in our study.

Changes in VRD in our diabetic patients were strongly associated with the presence of $\mathrm{CAD}$. We have previously reported that non-diabetic patients with $\mathrm{CAD}$ have up to a 1.5fold increase in VRD compared to healthy controls ${ }^{7}$. Furthermore, beat-to-beat QT interval variability has been associated with myocardial ischemia in coronary patients ${ }^{6}$. In large 
population-based studies, the presence of altered ventricular repolarization, measured with JTc interval, was predictive of incident coronary heart disease ${ }^{12}$. The combined results of these studies demonstrate that the presence of CAD can alter ventricular repolarization in various clinical settings.

The impact of CAD on ventricular repolarization in diabetes is not well known. In a large population-based study, the prevalence of CAD was related to the QT interval in patients with newly diagnosed type 2 diabetes ${ }^{13}$. In patients with type 1 diabetes, an increased QT dispersion was associated with ischemic heart disease and increased diastolic blood pressure, but not with neuropathy ${ }^{14}$. Similarly, in type 2 diabetes, the presence of CAD was related both to prolonged QTc interval and increased QT interval dispersion ${ }^{15}$. However, the QTc interval is significantly affected by neuroendocrine and metabolic factors, inflammation, and changes in sympathetic nervous activity. ${ }^{16,17}$ Therefore, QTc prolongation seems to be a marker of advanced disease rather than a simple reflection of ventricular repolarization instability. Although QT dispersion measurements have been proposed as an alternate risk marker in various clinical settings, their clinical use may be suspect as well as limited by poor reproducibility. ${ }^{18}$

VRD, measured by beat-to-beat QT interval variability, does not correlate with QTc interval duration and QT dispersion ${ }^{7,16}$. Therefore, the results of our study may offer new insights into the pathophysiology of abnormal ventricular repolarization in patients with type 2 diabetes. Though the underlying mechanism of ventricular repolarization changes is not fully understood in these patients, it seems related to the presence of CAD. Considering the importance of ventricular repolarization changes in the pathogenesis of ventricular arrhythmias, we suggest that VRD measurements are a simple, non-invasive adjunct in the risk stratification of patients with type 2 diabetes. 
References

1 Linnemann B, Janka HU. Prolonged QTc interval and elevated heart rate identify the type 2 diabetic patient at high risk for cardiovascular death. The Bremen Diabetes Study. Exp Clin Endocrinol Diabetes 2003;111:215-22.

2 Okin PM, Devereux RB, Lee ET, et al. Electrocardiographic repolarization complexity and abnormality predict all-cause and cardiovascular mortality in diabetes: The Strong Heart Study. Diabetes 2004;53:434-440.

3 Cardoso CR, Salles GF, Deccache W. QTc interval prolongation is a predictor of future strokes in patients with type 2 diabetes mellitus. Stroke 2003;34:2187-94.

4 Valensi PE, Johnson NB, Maison-Blanche P, et al. Influence of cardiac autonomic neuropathy on heart rate dependence of ventricular repolarization in diabetic patients. Diabetes Care 2002;25:918-923.

5 Dekker JM, Feskens EJ, Schouten EG, et al. QT duration is associated with levels of insulin and glucose intolerance. The Zutphen Elderly Study. Diabetes 1996;45:376-380.

6 Murabayashi T, Fetics B, Kass D, et al. Beat-to-beat QT interval variability associated with acute myocardial ischemia. J Electrocardiol 2002;35:19-25.

7 Vrtovec B, Starc V, Starc R. Beat-to-beat QT interval variability in coronary patients. J Electrocardiol 2000;33:119-125.

8 Task force of the European society of cardiology and the North American society of pacing and electrophysiology. Heart rate variability: Standards of measurement, physiological interpretation, and clinical use. Circulation 1996;93:1043-51.

9 Berger RD. QT variability. J Electrocardiol 2003;36 Suppl:83-7.

10 Weston PJ, Glancy JM, McNally PG, et al. Can abnormalities of ventricular repolarization identify insulin dependent diabetic patients at risk of sudden cardiac death? Heart 1997; 78:56-60. 
11 Ziegler D. Diabetic cardiovascular autonomic neuropathy: prognosis, diagnosis and treatment. Diabetes Metab Rev 1994;10:339-383.

12 Crow RS, Hannan PJ, Folsom AR. Prognostic significance of corrected QT and corrected JT interval for incident coronary heart disease in a general population sample stratified by presence or absence of wide QRS complex. The ARIC study with 13 years of follow-up. Circulation 2003;108:1985-89.

13 Festa A, D'Agostino R Jr, Rautaharju P, et al. Relation of systemic blood pressure, left ventricular mass, insulin sensitivity, and coronary artery disease to QT interval duration in nondiabetic and type 2 diabetic subjects. Am J Cardiol 2000;86:1117-22.

14 Veglio M, Giunti S, Stevens LK, et al. Prevalence of Q-T interval dispersion in type 1 diabetes and its relation with cardiac ischemia: the EURODIAB IDDM Complications Study Group. Diabetes Care 2002;25:702-7.

15 Veglio M, Bruno G, Borra M, et al. Prevalence of increased QT interval duration and dispersion in type 2 diabetic patients and its relationship with coronary heart disease: a population-based cohort. J Internal Medicine 2002;251:317-324.

16 Berger RD, Kasper EK, Baughman KL, et al. Beat-to-beat QT interval variability: novel evidence for repolarization lability in ischemic and nonischemic dilated cardiomyopathy. Circulation. 1997;96:1557-1565.

17 Darbar D, Fromm MF, Dellorto S, et al. Sympathetic activation enhances QT prolongation by quinidine. J Cardiovasc Electrophysiol. 2001;12:9-14.

18 Malik M, Batchvarov VN. Measurement, interpretation and clinical potential of QT dispersion. J Am Coll Cardiol. 2000;36:1749-1766. 


\section{Figure legend}

Figure 1: Ventricular repolarization dynamics in diabetic patients with, or without coronary artery disease $(\mathrm{CAD})$, and in the control group (Controls) 
Table 1: Baseline characteristics of subjects from the Study and Control groups

\begin{tabular}{lccc}
\hline & $\begin{array}{c}\text { Study Group } \\
(\mathbf{N}=\mathbf{3 8})\end{array}$ & $\begin{array}{c}\text { Control Group } \\
(\mathbf{N}=\mathbf{3 8})\end{array}$ & $\boldsymbol{P}$ \\
\hline Age [years] & $54 \pm 7$ & $53 \pm 8$ & NS \\
Male gender & $22(58)$ & $22(58)$ & NS \\
BMI [kg/m $\left.{ }^{2}\right]$ & $29.9 \pm 3.6$ & $30.9 \pm 5.2$ & NS \\
Total cholesterol [mmol/l] & $5.2 \pm 1.1$ & $4.9 \pm 1.3$ & NS \\
Potassium [mmol/l] & $4.5 \pm 0.4$ & $4.4 \pm 0.3$ & NS \\
Calcium [mmol/l] & $2.3 \pm 0.2$ & $2.4 \pm 0.1$ & NS \\
NS Magnesium [mmol//] & $2.3 \pm 0.3$ & $2.2 \pm 0.3$ & NS \\
Heart rate [bpm] & $74 \pm 15$ & $73 \pm 14$ & NS \\
Systolic BP [mmHg] & $131 \pm 21$ & $129 \pm 24$ & NS \\
Diastolic BP [mmHg] & $86 \pm 11$ & $82 \pm 8$ & NS \\
\hline
\end{tabular}

Values are presented as mean $\pm \mathrm{SD}$ or number (percent). $\mathrm{BMI}=$ body mass index; $\mathrm{BP}=$ blood pressure. 
Table 2: Multivariate analysis of potential predictors of increased ventricular repolarization dynamics in patients with diabetes

\begin{tabular}{lcc}
\hline & $\begin{array}{c}\text { Multivariate } \\
\boldsymbol{P} \text {-value }\end{array}$ & $\begin{array}{c}\text { Hazard ratio } \\
(\mathbf{9 5 \%} \mathbf{C I})\end{array}$ \\
\hline Presence of CAD & $0.008^{*}$ & $4.4(1.4-13.7)$ \\
Male gender & 0.23 & - \\
Age $>$ 60 years & 0.43 & - \\
Systolic BP $>\mathbf{1 3 0} \mathbf{~ m m H g}$ & 0.45 & - \\
\hline CAD = coronary artery disease; $\mathrm{BP}=$ blood pressure; ${ }^{*} P<0.05$.
\end{tabular}




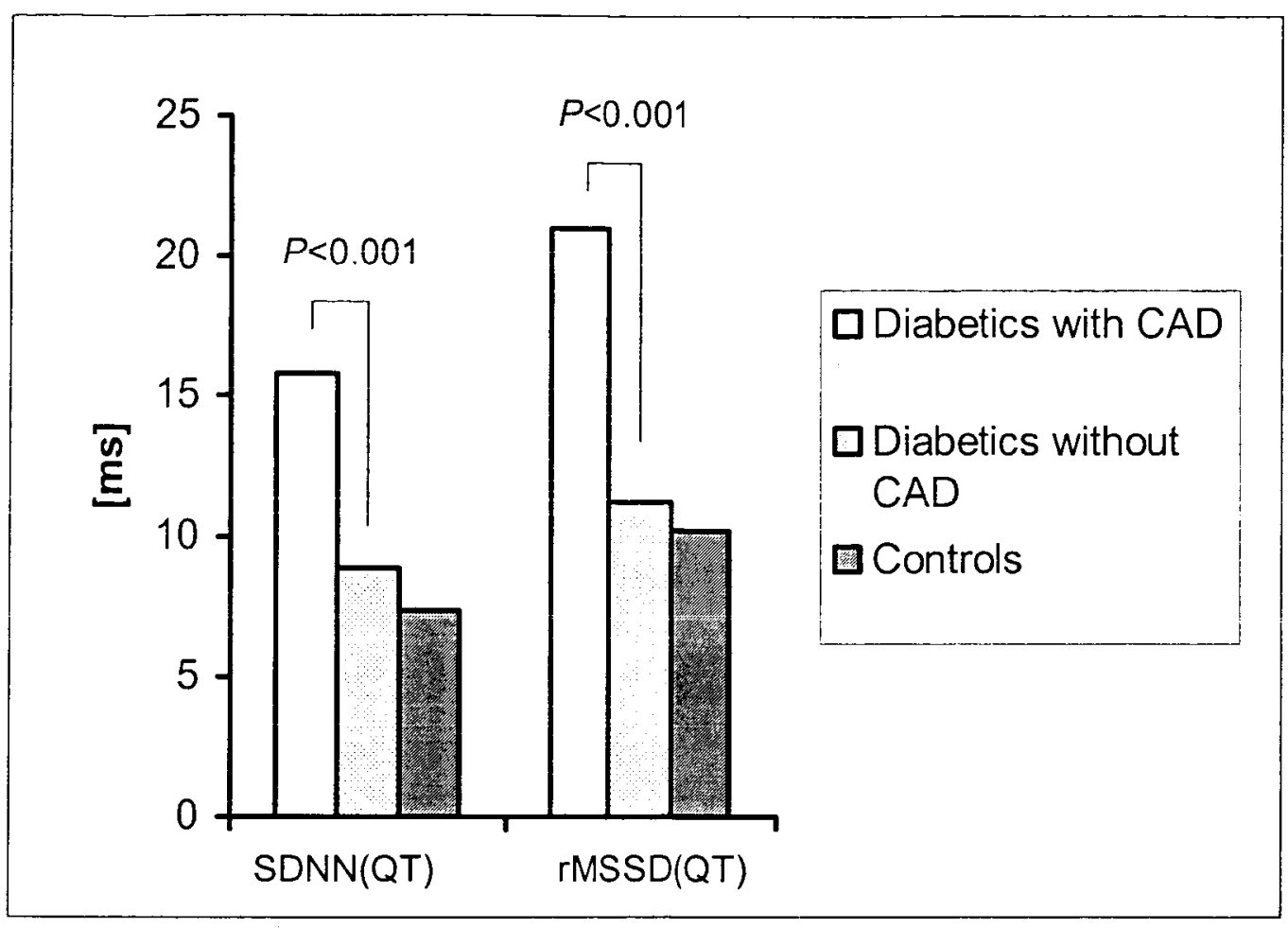

